\title{
Development of Smart Technology for Complex Objects Prediction and Control on the Basis of a Distributed Control System and an Artificial Immune Systems Approach
}

\author{
Samigulina Galina ${ }^{1}$, Samigulina Zarina ${ }^{2, *}$ \\ ${ }^{1}$ Intellectual control systems and forecasting, Institute of information and computing technologies, 050010, Kazakhstan \\ ${ }^{2}$ Faculty of Information Technologies, Kazakh British Technical University, 050000, Kazakhstan
}

A R T I C L E I N F O

Article history:

Received: 07 February 2019

Accepted: 03 May, 2019

Online: 21 May, 2019

Keywords :

Intellectual control systems

Complex object

Artificial Immune System

Distributed control system

Multi-agent Smart system

\begin{abstract}
A B S T R A C T
This paper is an extension of work originally presented in 2018 Global Smart Industry Conference (GloSIC). Researches are devoted to the development of Smart technology for complex objects control and prediction on the basis of a distributed Honeywell DCS control system of the TengizChevroil enterprise using the example of a technological process of medium pressure gas cleaning. The article describes how on the basis of the multialgorithm approach there was developed a modified algorithm based on modern artificial intelligence methods in order to select informative features (principal component method, Random Forest algorithm, particle swarm algorithm) and artificial immune systems (clonal selection) solving the image recognition problem and predicting the state of a complex control object. There was conducted a comparative analysis of the simulation results using the example of real production data (daily data of sensors from an average pressure absorber).
\end{abstract}

\section{Introduction}

Nowadays, the efficiency of industrial enterprises depends on many factors: material and technical support (installation of modern production equipment, modernization and reconstruction of the existing technical base), economic factors (growth of production and raw materials costs), management factors (information support for decision-making), and etc [1]. Therefore, modern researches in the field of development of automated complex objects control systems are aimed at increasing the reliability and at reducing the influence of various negative factors on the production process.

The implementation of modern Smart technology enables the possibility to improve production by effective prediction of the technical state of the equipment and the timely resolution of problems at the stage of their occurrence in real time. A characteristic feature of the complex objects control is the need to process a huge array of production data, as well as prediction and decision-making under the condition of parameters uncertainty.

In order to solve such problems at the complex objects control process, there were proven modern methods of artificial intelligence with the following advantages: the ability to process

${ }^{*}$ Corresponding Author Zarina Samigulina, e-mail: zarinasamigulina@mail.ru www.astesj.com

https://dx.doi.org/10.25046/aj040312 multidimensional data, self-organization and relative ease of implementation.

Widespread use has control systems based on neural networks $(\mathrm{NN})$. For example, in [2] there is presented the use of a decentralized robot control system based on a neural network. There were proposed four decentralized control systems which can identify the dynamics of the robot's movement. Training of each neural network is performed online using the advanced Kalman filter. The researches [3] are devoted to the development of a combined adaptive neural network and to the nonlinear prediction model for production processes control.

The effectiveness of the proposed method was tested on a system of reactors with continuous mixing. In work [4] there is presented a PID controller based on a neural network for the $\mathrm{pH}$ neutralization process control system. The classical PID control cannot adapt to changes in the process, and therefore a PID controller with a signal neuron was developed. The simulation results showed the reliability and adaptability of the proposed control system.

In addition to neural networks there are used swarm intelligence (SI) algorithms for complex objects control. For example, in work [5] there is presented the application of the 


\section{G.A. Samigulina et al. / Advances in Science, Technology and Engineering Systems Journal Vol. 4, No. 3, 75-87 (2019)}

particle swarm algorithm (PSO) for the optimal choice of process control parameters. In researches [6] there was used an ant colony optimization at setting the PID controller for a conical tank. The proposed approach has shown better results compared to setting of a closed loop based on the Ziegler-Nichols method and to setting of a controller in a closed loop system. In work [7] there is presented the development of a control system for autonomous mobile robots based on a bee colony algorithm and a genetic algorithm. Researches [8] are devoted to applying the method of bacteria movement optimization at the development of a PID controller by a two-wheeled robot with a two-way movement mechanism for a working environment with limited space.

Also there is widely used an approach based on fuzzy logic for complex objects control. Another study a control system for mobile robots with nonholonomic wheels moving on a plane on the basis of a fuzzy controller in order to achieve a given trajectory of motion. The problem of rounding obstacles is considered. Parameters of PID controller are calculated on the basis of fuzzy logic. There are presented simulation results based on a real vehicle. The researches are devoted to cascade control based on a fuzzy PID controller for a conveyor for weighing materials. The article presents a non-linear model of a conveyor system of continuous weighing, taking into account the delay time of material transportation. There has been developed a PID controller based on fuzzy logic with two control loops: an internal speed control loop and an external mass flow control loop. The results of simulation and experiment show that the proposed algorithm for fuzzy PID control can improve the quality of material mass flow control in an industrial conveyor system of continuous weighing using a programmable logic controller.

In work [9] there is proposed an innovative approach for fuzzy control systems setting with low parametric sensitivity based on the Gray Wolfs Optimization Algorithm (GWO). The servo system is controlled on the basis of the Takagi-Sugeno-Kang fuzzy PI controller (TSK PI-FC). The GWO algorithm is used in solving the optimization problem. Article [10] is devoted to an optimized fuzzy-genetic algorithm (F-GA) for controlling the process of production of aluminum foam with open pores. The application of the F-GA method allows to take into account the uncertainty associated with the model and the statistical variability of the production process within one model.

Nowadays, the researches in the field of the use of the bioinspired approach of artificial immune systems (AIS) for complex objects control are also promising. In work [11] there is presented AIS application at PID controller parameters setting for nonlinear control systems. Researches [12] are devoted to the development of a complex object control algorithm based on an adaptive artificial immune system. The simulation was carried out on the example of a reactor with a mixing function. The article [13] provides an AIS application for control a group of robots. The work [14] is devoted to the use of a meta-heuristic AIS for signal evaluation in identifying the contour of the angle control of an unmanned aerial vehicle. In the article [15] there is used the AIS algorithm for preliminary signal processing at detection of failures for autonomous underwater vehicles. Article [16] is devoted to applying the AIS approach (negative selection algorithm) in order to control the combustion process in the chamber with the implementation on the microcontroller. In researches [17] there was developed an immune reconfigurable controller based on four modules for the immune system modeling: the observation process, response, mechanism of memory and self-training. The proposed algorithm is able to detect drive failures and to provide failuretolerant control for the systems with multiple inputs and outputs.

In addition to bioinspired methods, machine learning algorithms for complex objects control and solving the problem of identifying informative features have become widespread. The most promising is the Random Forest (RF) algorithm. For example, in [18], RF is used to detect defective plates by chemical characteristics in the production of semiconductors. The article [19] presents the use of algorithms for machine learning of RF, decision trees, etc. in heavy industry for analysis of large amounts of production data. The work [20] is devoted to the use of the Random Forest algorithm in sensor failures diagnostics. The algorithm is used to reduce memory requirements in the process of assessing the state of the equipment in real time.

Therefore, the analysis of the literature proves the relevance of the development of modern Smart technologies and prediction of complex objects control based on industrial enterprises using artificial intelligence methods.

The following structure of the article is proposed. The second section contains the formulation of the research problem. The third section presents the development of Smart technology for complex objects control and prediction based on the Honeywell Experion Process Knowledge System (PKS) distributed control system (DCS) and the approach of artificial immune systems; reviewed the operation of the DCS at the Tengizchevroil enterprise, and presented the general structure of the Smart technology. The fourth section is devoted to the development of a modified algorithm of artificial immune systems. The fifth section describes the multiagent Smart system for complex object prediction and control under industrial operation conditions. The sixth section contains the simulation results. Description of a real complex mediumpressure absorber, its principle of operation, readings from sensors, etc. The simulation results are presented in the form of graphs, as well as a comparative analysis of the effectiveness of the proposed algorithms. The conclusion describes the main findings and a list of references.

\section{Problem statement}

Since the complex objects control with the presence of parameters uncertainty in the conditions of industrial operation is a time-consuming task due to the need to process multidimensional data in real time, the use of promising heuristic algorithms of artificial intelligence is important.

The research problem statement is formulated as follows: it is necessary to develop a modern Smart technology for complex industrial objects control based on a multi-algorithm approach. Within the framework of the multi-algorithm approach to synthesize a modified algorithm of artificial immune systems in order to solve the problem of prediction and control. In order to organize an autonomous, flexible and decentralized control system based on the proposed technology, it is necessary to develop a multi-agent Smart system for implementation in real production based on TengizChevroil enterprise, with the aim of working with Honeywell PKS distributed control systems. 


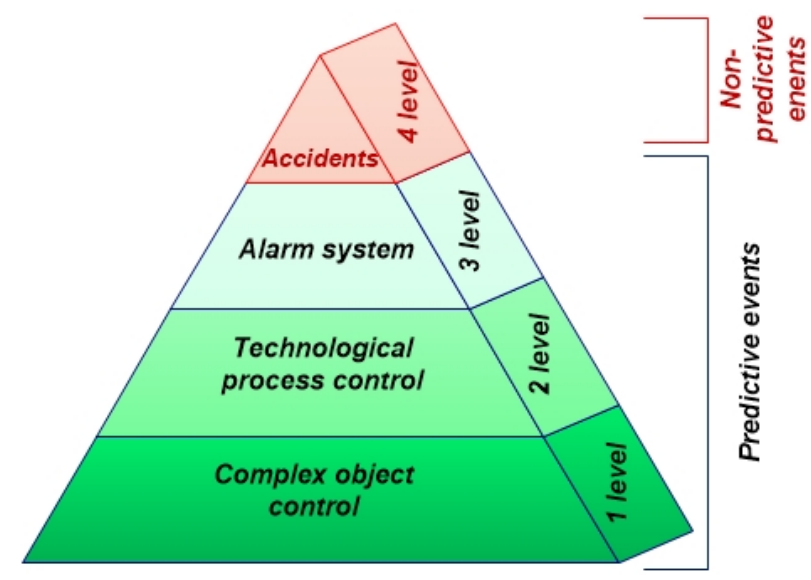

Figure 1: Honeywell DCS process control system with independent protection levels

\section{Development of Smart technology for complex objects prediction and control on the basis of a distributed control system and an artificial immune systems approach}

\subsection{Distributed control system at TengizChevroil enterprise}

TengizChevroil is the largest company engaged in the development and production of oil in the territory of the Republic of Kazakhstan together with the American company Chevron. In TengizChevroil there is used modern industrial equipment from Honeywell, in particular, Honeywell PKS distributed control systems (DCS). Nowadays, the Experion PKS system is the best in the field of distributed control systems and received an award from the magazine "Control Engineering". The company created a consortium for emergency situations control, aimed at exploring the causes of emergency situations and developing solutions aimed at reducing them. Therefore, the researches on improving the quality of equipment at the enterprise are relevant.

The Honeywell PKS distributed control system contains several independent levels of protection (Figure 1), designed to work with predictable and unpredictable events [23].

Predicted events include the following levels of independent protection:

1 level. Maintains "normal" operating conditions of the process. The distributed control system is engaged in the collection and processing of technological parameters, as well as their adjustment to ensure a given mode.

2 level. This level requires decision-making from the operator if the alarm is triggered and the first level is unable to maintain normal operating conditions of the control object.

3 level. This level indicates that the control object has left the "normal conditions" of operation mode and a controlled stop is required. The Safety Instrumented Systems (SIS) is activated. The SIS security system also includes: Emergency Shutdown System (ESD), Fire \& Gas System (F\&G), Safety Shutdown System (SSD) и т.д.
4 level. The level refers to unpredictable events, such as the occurrence of fires, gas leaks and other major accidents. A distributed system cannot predict the occurrence of such accidents, but it is designed for quick response and minimization of damage.

Information on the operation of the technological process is stored in the historical module (History Module, HM) of Honeywell DCS [23]. Developed Smart technology is aimed at working with the predicted events of the Honeywell DCS, with obtaining data for processing using the HM.

\subsection{Smart technology based on Honeywell PKS distributed control system}

Let consider the general structure of the proposed Smarttechnology for complex objects prediction and control. The Honeywell PKS distributed control system consists of nodes described by controllers, operator consoles, control devices, data storage equipment, etc. The information transfer between nodes occurs through a local and universal control network. Information is collected in a historical module that is connected to a local control network, and a process history database is formed here.

The developed Smart-technology is designed to work with the predicted events of Honeywell PKS (Figure 1). The process history database containing current information on the behavior of a complex control object (readings from sensors, monitoring systems, etc.) is processed on the basis of a multi-algorithm approach (Figure 2).

Then a database of optimal parameters is formed for prediction and control on the basis of AIS algorithm, clonal selection. Depending on the nature of the processed data in the modified algorithm based on the AIS method, the most effective algorithm is selected that shows the best predictive result.

Further in the paper, as an example, we will consider the complex control object of the Tengizchevroil enterprise: a fragment of the Installation 300 for cleaning gases from acidic components [21]. 


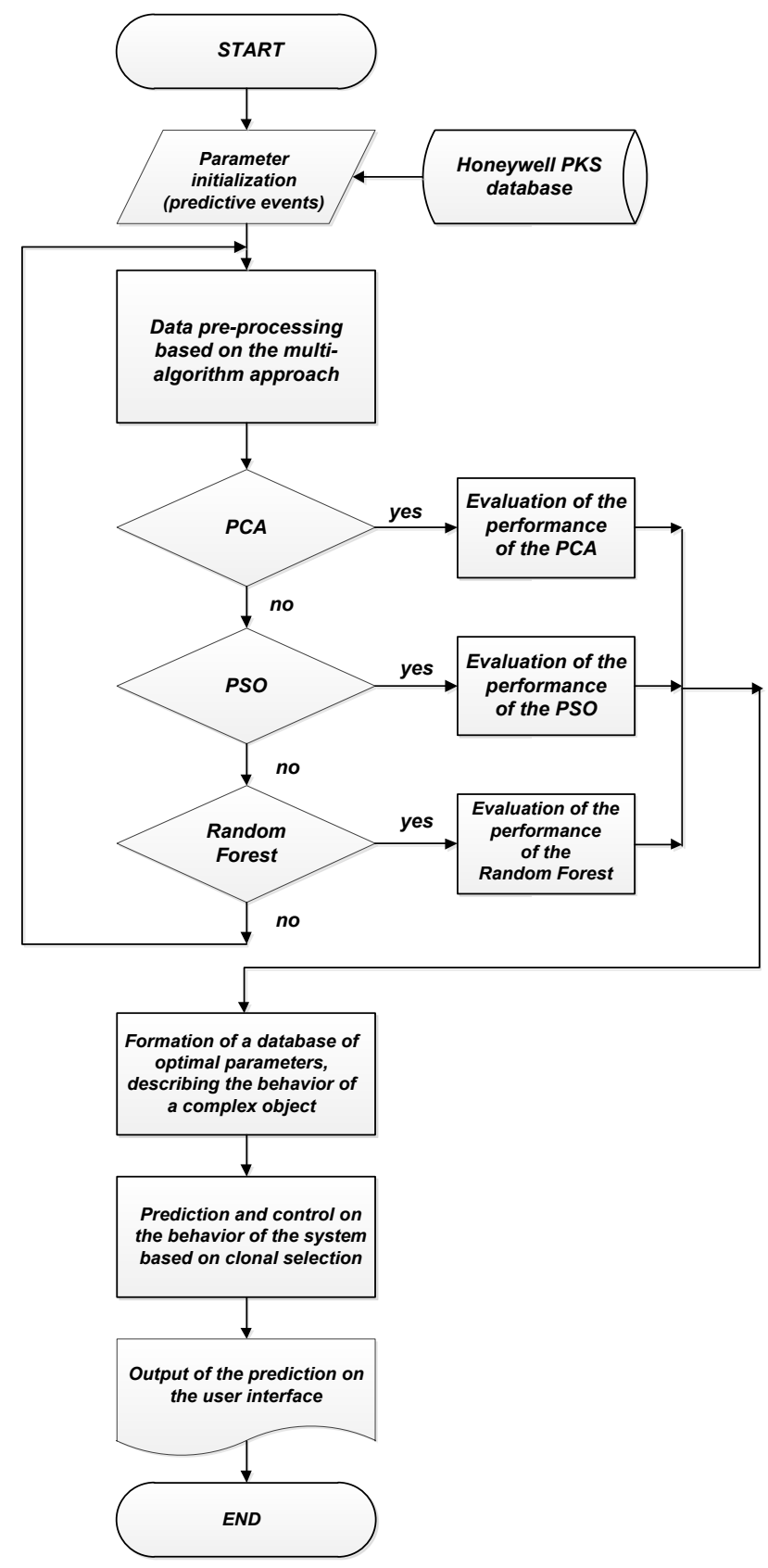

Figure 2: The structure of the developed Smart technology for complex objects prediction and control based on Honeywell PKS

\section{Modified artificial immune algorithm based on clonal selection}

Nowadays, the approach of artificial immune systems is a promising area of research. This method has the following advantages: adaptability, memory, dynamic arrangement of elements, ability to self-organization, etc. There are several main areas of AIS research: negative selection algorithms (NSA), immune system algorithms (INA), danger theory algorithms (DTA), and clonal selection algorithms (CSA) [22].

Let consider in more detail the clonal selection algorithm [2325]. When a foreign antigen enters the body, special cells Blymphocytes produce antibodies $A_{b}$. Each cell produces only one type of antibody that is relatively specific for the antigen. At binding antibodies (receptors), as well as using T-helper cells, the antigen stimulates B cells to multiply and to mature into plasma cells. Different cell divisions produce clones, by which there is meant a set of cells that are descendants from a single cell. Plasma cells most actively produce antibodies B-lymphocytes that divide rapidly. Antibodies $A_{b}$ divide much more slowly. Lymphocytes can also differentiate in addition to plasma cells into long-lived memory cells - B - memory cell. Memory cells circulate through the body through the blood, lymph and tissue. In a secondary immune response, they can turn into large lymphocytes capable of producing antibodies with high affinity. These cells are preselected for the specific antigen that stimulated the primary response. The clonal selection algorithm is based on the following operations: selection, cell cloning and mutation. The selection procedure is carried out for cells with higher affinity and subsequent cloning, while other cells undergo a mutation process, which helps to improve their quality.

The clonal selection algorithm is presented as follows [23]:

\section{Algorithm 1}

Step 1. Generation of a set of possible solutions $P$, composed of a subset of memory cells $M$, added to the remaining population $P_{r}$.

$$
P=P_{r} \cdot M
$$

Step 2. Determination of the best individuals $n$, the population $P_{n}$ based on the value of affinity (affinity).

Step 3. Cloning of $n$ best individuals of a population, with the generation of a temporary population of clones $C$.

Step 4. Representation of a clone population to a hypermutation procedure, where hypermutation is proportional to the affinity of the antibody and antigen. Creation of a population of mature antibodies $C^{*}$.

Step 5. Repeated selection of improved individuals from the population $C^{*}$ in order to form a set of memory $M$. Some members of the population $P$ may be replaced by other improved population individuals $C^{*}$.

Step 6. Replacement of antibodies $d$ by the new ones. Cells with lower affinity have a higher probability of replacement.

Modern methods of artificial intelligence are well established for the development of complex objects control systems, but unfortunately these approaches are not universal, and need to be customized for specific cases, taking into account the features of the object and the system parameters. The quality of forecasting and management directly depends on the processed data. The higher the quality of the data is, the more accurate is the forecast. Therefore, the application of the multi-algorithm approach is actual, within the framework of which the procedure of preliminary production data processing is carried out on the basis of modern statistical and intellectual algorithms. As a result of 


\section{G.A. Samigulina et al. / Advances in Science, Technology and Engineering Systems Journal Vol. 4, No. 3, $75-87$ (2019)}

research several methods are considered. Algorithms that will give the best predictive result are used for further research. Preliminary production data processing within the framework of the proposed Smart technology is carried out on the basis of the following approaches:

- Statistical approach - factor analysis and principal component method;

Statistical approaches are often used to process production data. In order to solve the problem of reduction of noninformative parameters during the study of complex industrial facilities, factor analysis (principal component method) has proven itself well. Among the advantages of factor analysis we can note the ability to analyze hidden (latent) relationships between parameters, as well as the relative simplicity of implementation [26]. As a disadvantage there should be noted the difficulties in the interpretation of the obtained results.

The algorithm of factor analysis consists of the following steps. The entire data set must be rotated counterclockwise, with the first axis associated with the maximum dispersion, and each subsequent one with the others. According to the results of the data rotation, the parameters that lie closer to the origin of coordinates are subject to reduction, as uninformative.

- Swarm intelligence - particle swarm optimization algorithm;

Let consider the concept of swarm intelligence (SI) [27]. The term SI was proposed by Beni and Wang in application to cellular robots and implied the collective behavior of a decentralized selforganizing system. Then it was used in the theory of artificial intelligence and describe the social behavior of living organisms (particle swarm method, ant algorithm, bee algorithm, wolf pack algorithm, bat algorithm, etc.).

In the proposed Smart technology, in the framework of the multi-algorithm approach, a particle swarm optimization algorithm is considered in detail.

A feature of the PSO method is that during each iteration, the solution is evaluated using the objective function to determine its suitability [28]. Each solution is represented as a particle in the search space. Particles move in the search space in order to find the maximum value of the objective function. As the particles move, each particle has a position in the search space, speed, and an individual best position. The PSO algorithm consists of the following steps [26, 27]:

\section{Algorithm 2 Particle Swarm Optimization}

Step1 . Initialization of a particles swarm.

Step 2. Evaluation of the suitability of each particle.

Step 3. Calculation of historically optimal positions of individuals.

Step 4. Calculation of historically optimal positions of the swarm.

Step 5. Speed and position update of particles according to the equation of speed and position. Repeat of this iteration until the stop condition is reached.
The speed of each particle is calculated by the formula:

$$
\begin{aligned}
& v_{i}(t+1)=\varpi v_{i}(t)+c_{1} \cdot r_{1} \cdot\left(\hat{x}_{i}(t)-x_{i}(t)\right)+ \\
& +c_{2} \cdot r_{2} \cdot\left(g(t)-x_{i}(t)\right)
\end{aligned}
$$

where $i$ - particle index; $\varpi$-inertia coefficient $0.8 \leq \varpi \leq 1.2$; $c_{1}, c_{2}$ - acceleration coefficients $\left(0 \leq c_{1}, c_{2} \leq 2\right) ; r_{1}, r_{2}$ - random values lying in the range $0 \leq r_{1}, r_{2} \leq 1 ; v_{1}(t)$-particle speed in time $t ; x_{1}(t)$-_particle position in time $t ; g(t)$ - the best swarm solution in time $t$.

The change in the position of each particle of the swarm is calculated by the following formula:

$$
x_{i}(t+1)=x_{i}(t)+v_{i}(t+1)
$$

The advantages of the PSO algorithm include: a simple implementation; small amount of adjustable parameters; parallel computations are possible; with a certain setting has the ability to fast convergence; no matches and mutations; has small requirements for computer memory and fast calculation speed; can be used in case when the representation of a mathematical model is impossible.

- Random Forest (RF) algorithm, etc.

Nowadays, the RF algorithm is a promising method of machine learning, capable of solving the problems of image recognition, prediction and selection of informative features [28]. A random forest is created on the basis of decision trees and has the same set of hyperparameters, while in RF the process of searching for the root node and the separation of object nodes is performed randomly $[29,30]$. The more trees in the forest, the more accurate the simulation result are.

The advantages of the RF algorithm are following: there is no risk of retraining of the model; the algorithm has flexibility and high accuracy; no need to scale data; individual decision trees can be trained in parallel; data processing ability with a large amount of attributes, etc.

The operation of the Random Forest algorithm is presented below:

\section{Algorithm 3 Random Forest}

Step 1. Arbitrary selection of $f$ signs from the total number of $F$ signs.

Step 2. Calculation of a node among $f$ features using the best separation point.

Step 3. Separate a node on child nodes using the best partition.

Step 4. Repeat steps 1-3 until reaching the value of the number one.

Step 5. Creation of a forest by $n$ repeating of the steps 1-4 in order to create the $n$ number of trees.

Based on the previously discussed methods, the modified AIS algorithm based on clonal selection can be described as follows (Figure 2). 
Algorithm 4 Modified artificial immune algorithm

1. Connection to the historical module of the Honeywell PKS distributed system.

2. Data collection (readings from sensors) describing the behavior of a complex control object.

3. Formation of a database of system parameters.

4. Pre-processing of the obtained database, rationing, centering and filling in the missing data.

5. Formation of the optimal set of parameters due to the reduction of non-informative data based on the multi-algorithm approach.

5.1 Reduction of non-informative parameters based on the statistical approach, the principal component method.

5.1.1 Evaluation of the performance of the PCA algorithm.

5.1.2 Formation of a database of optimal parameters DB_PCA, describing the behavior of a complex object.

5.2 Reduction of non-informative parameters based on swarm intelligence, particle swarm algorithm.

5.2.1 Evaluation of the performance of the PSO algorithm.

5.2.2 Formation of a database of optimal parameters DB_PSO, describing the behavior of a complex object.

5.3 Reduction of non-informative parameters based on the Random Forest algorithm.

5.3.1 Evaluation of the performance of the Random Forest algorithm.

5.3.2 Formation of a database of optimal parameters DB_RF, describing the behavior of a complex object.

6. The solution of the problem of image recognition on the basis of clonal selection. Processing the received databases (DB_PCA, DB_PSO, DB_RF) and prediction.

6.1 Selection of an efficient algorithm that shows the best prognostic result.

6.2 Decision-making on the complex object control.

The proposed technology in industrial operation is most conveniently implemented on the basis of a multi-agent approach. Further there is presented a developed multi-agent Smart system for complex objects prediction and control.

\section{Multi-agent object control Smart-system}

Nowadays, multi-agent systems (MAS) are widely used in the field of industrial automation and artificial intelligence. A multiagent system consists of independent agents with autonomy, decentralization, and flexibility. At developing complex industrial systems using MAS, the process of modeling and control is greatly facilitated. The paper [31] presents the MAS technology for organization of a flexible production process of customized products, through cooperation between companies, for the integration of heterogeneous production systems. Article [32] presents the use of a multi-agent artificial immune system for detecting and classifying network intrusions. Researches [33] are devoted to a decentralized multi-agent control system based on artificial immune systems. This system consists of the following target agents: the agent of self-organization, the agent of interaction, the agent of following specific goals and has the properties of flexibility, the ability of agents to work autonomously, the ability to optimally distribute the system resource, multifunctionality and self-organization.
Based on the foregoing, the development of a multi-agent Smart system for complex objects control at TengizShevroil for the integration of the developed modified artificial intelligence algorithms into production is relevant. Figure 3 shows the structure of the multi-agent Smart system.

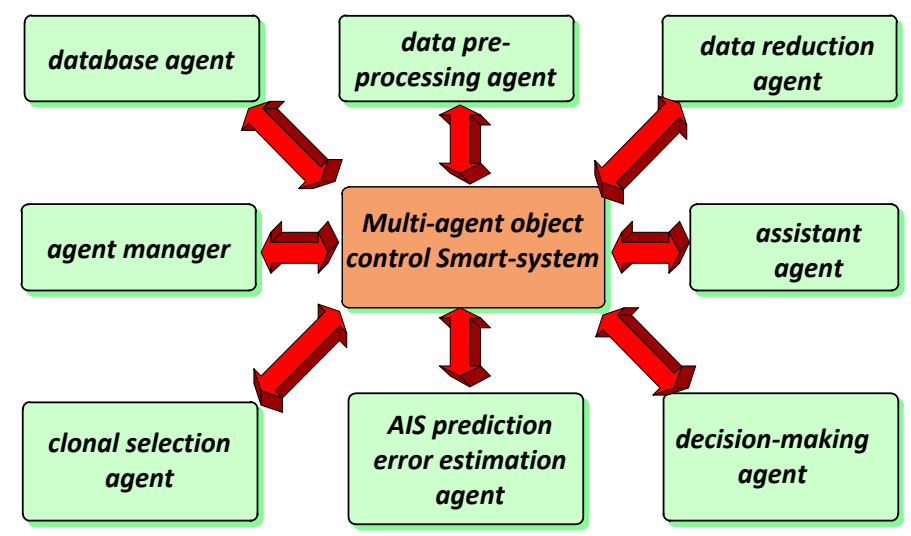

Figure 3: Structure of multi-agent smart system for complex objects control

Table 1 describes the main agents and their functions.

Table 1: Functions of agents of multi-agent smart system

\begin{tabular}{|c|c|}
\hline Agent name & Function of the agents \\
\hline $\begin{array}{l}\text { Database } \\
\text { agent }\end{array}$ & $\begin{array}{l}\text { A database agent is needed to organize data } \\
\text { readout from the Honeywell PKS historical } \\
\text { module of the distributed control system. The } \\
\text { generated database consists of sensors and } \\
\text { monitoring systems, which allow to assess the } \\
\text { state of the technological control object } \\
\text { (normal operation, work with deviations, an } \\
\text { accident, etc.). }\end{array}$ \\
\hline $\begin{array}{l}\text { Data } \\
\text { preprocessing } \\
\text { agent }\end{array}$ & $\begin{array}{l}\text { The agent of preliminary data processing is } \\
\text { responsible for rationing, centering, filling in } \\
\text { the missing data in the database of parameters } \\
\text { of the control object. The agent interacts } \\
\text { directly with the database agent. }\end{array}$ \\
\hline $\begin{array}{l}\text { Data } \\
\text { reduction } \\
\text { agent }\end{array}$ & $\begin{array}{l}\text { The data dimension reduction agent performs } \\
\text { information processing based on the multi- } \\
\text { algorithm approach for reducing non- } \\
\text { informative parameters describing the } \\
\text { behavior of a complex object. }\end{array}$ \\
\hline $\begin{array}{l}\text { Clonal } \\
\text { selection } \\
\text { agent }\end{array}$ & $\begin{array}{l}\text { The agent implements the approach of } \\
\text { artificial immune systems, the clonal selection } \\
\text { algorithm for prediction of the behavior of a } \\
\text { complex object. }\end{array}$ \\
\hline $\begin{array}{l}\text { AIS } \\
\text { prediction } \\
\text { error } \\
\text { estimation } \\
\text { agent }\end{array}$ & $\begin{array}{l}\text { This agent is designed to select the algorithm } \\
\text { with the best predictive result. }\end{array}$ \\
\hline $\begin{array}{l}\text { Decision- } \\
\text { making agent }\end{array}$ & $\begin{array}{l}\text { The decision support agent generates } \\
\text { recommendations for process control based on } \\
\text { the obtained data on the results of the }\end{array}$ \\
\hline
\end{tabular}


G.A. Samigulina et al. / Advances in Science, Technology and Engineering Systems Journal Vol. 4, No. 3, 75-87 (2019)

\begin{tabular}{|l|l|}
\hline & $\begin{array}{l}\text { prediction based on the modified AIS } \\
\text { algorithm. }\end{array}$ \\
\hline $\begin{array}{l}\text { Assistant } \\
\text { agent }\end{array}$ & $\begin{array}{l}\text { The agent contains reference information } \\
\text { about the algorithms and principles of data } \\
\text { processing. Assistant functions are necessary } \\
\text { for correct input of information into the } \\
\text { system. }\end{array}$ \\
\hline $\begin{array}{l}\text { Agent } \\
\text { manager }\end{array}$ & $\begin{array}{l}\text { Agent Manager coordinates the work of other } \\
\text { agents. }\end{array}$ \\
\hline
\end{tabular}

\section{Simulation results}

\subsection{Technological process of oil gases cleaning from acidic components at installation 300}

Researches were conducted on the real production data of the TengizChevroil company. The enterprise consists of several complex technological lines (CTL) and installations (Figure 4).

As the control object we will consider the Installation 300, which is intended for the petroleum gases cleaning from acid components: hydrogen sulfide, carbon dioxide and carbon dioxide. Acidic components are removed using a solution of diethanolamine [21]. The raw materials of the U300 are high and medium pressure oil gases coming from Installation 200 (U200) intended for gas separation. The final products of the U300 are: purified high-pressure gas entering the Installation 700 for fractionation; refined medium pressure gas returned to the U200 for compression; acid gas with a high content of hydrogen sulfide, is sent to the installation 400 for the production of sulfur. In general, the U300 consists of the following main components: highpressure gas cleaning, medium-pressure gas cleaning, dietalomine regeneration, amine filtration, chemical supply, etc.

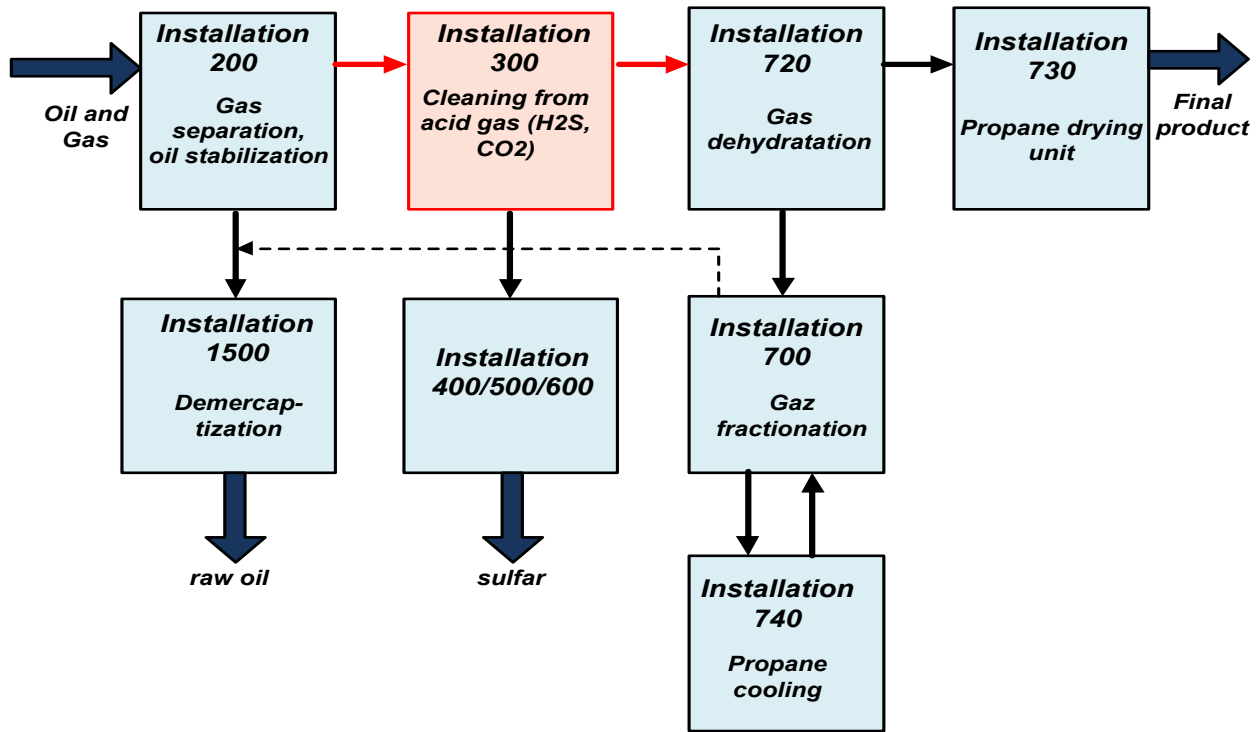

Figure 4: The structure of the Tengizchevroil enterprise

Table 2: Specification of medium pressure gas cleaning equipment

\begin{tabular}{|l|l|}
\hline $\begin{array}{c}\text { Designation on the } \\
\text { diagram }\end{array}$ & \multicolumn{1}{c|}{ Unit Name } \\
\hline D-302 & Medium Pressure Absorber \\
\hline F-328 & Medium Pressure Separator and Coalescer \\
\hline F-324 & Medium Pressure Filter-Separator \\
\hline F-320 & Separator \\
\hline F-319 & Separator \\
\hline F-303 & Saturated Amine Tank \\
\hline F-203 & Low pressure separator \\
\hline GC-200 & First stage line \\
\hline GC-201 & Second stage suction line \\
\hline EA-302 & Regenerator reboolers (4 heat exchangers) \\
\hline EA-304 & Regenerated / saturated amine heat exchanger \\
\hline
\end{tabular}




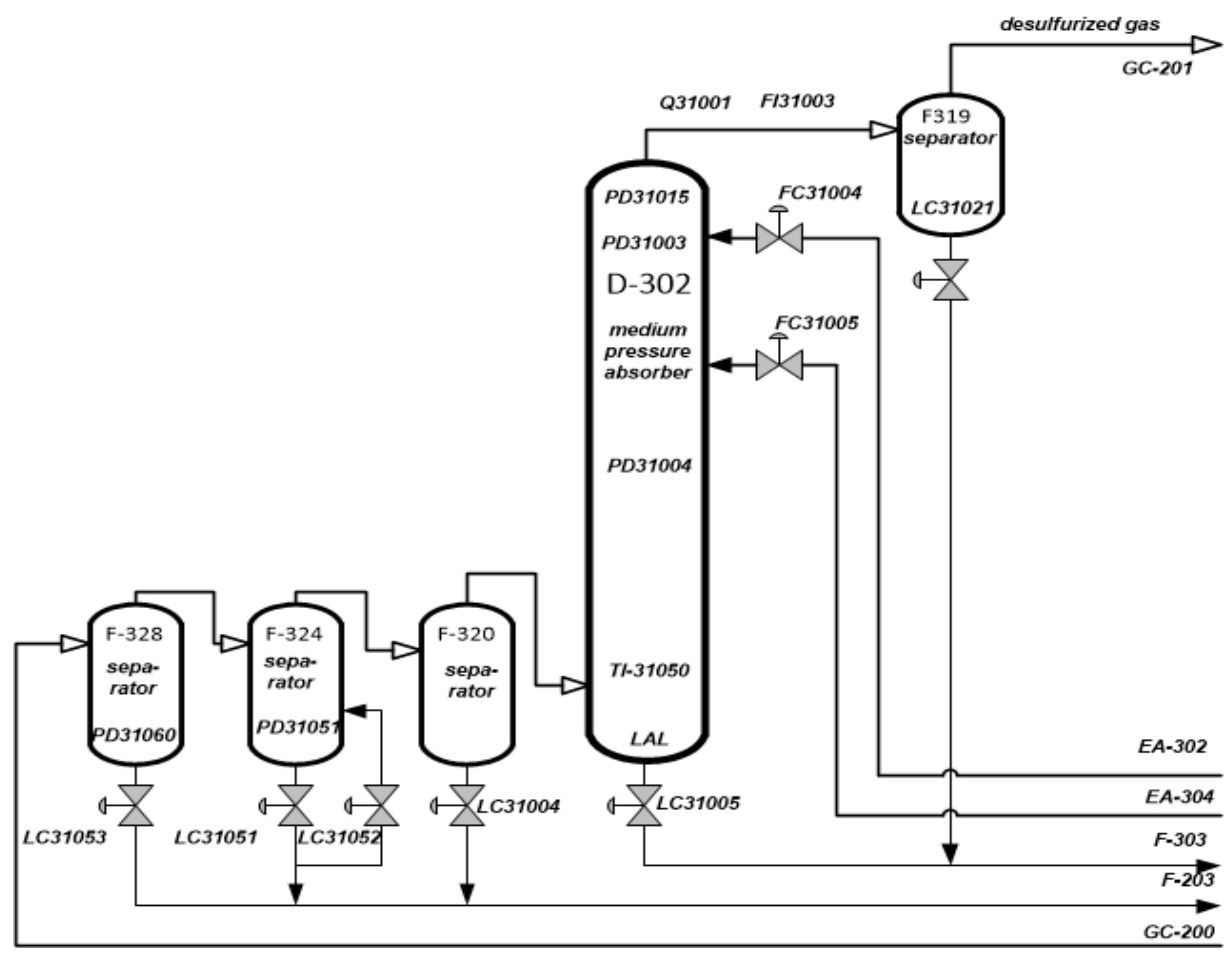

Figure 5: Fragment of the U300 technological scheme, medium pressure gas cleaning

The technological process of medium pressure gas cleaning in the absorber D-302 (medium pressure absorber) was chosen as a complex control object.

\subsection{Medium Pressure Gas Cleaning of the Installation 300}

Let consider a fragment of the U300, a medium pressure gas cleaning process (Figure 5, Table 2). The flow of raw gas enters the medium pressure absorber D-302 from the U200. In separators F-328, F-324, F-320 the hydrocarbon condensate is removed from the raw gas and transferred to the low-pressure separator F-203. This process is controlled by level controllers: LIC31053, LIC31051, LIC31052 and LIC31004.

Next, gas from the F-320 enters D-302. In the medium pressure absorber, the absorption process of $\mathrm{H}_{2} \mathrm{~S}$ and $\mathrm{CO}_{2}$ also takes place due to the contact with two streams of the solution of regenerated diethanolamine [21], the temperature of which should not be lower than $540^{\circ}$ in order to prevent condensation of hydrocarbons. In a medium pressure absorber, a pressure is approximately 24.5 bar. Gas temperature is measured using TI31050.

The absorber D-302 in the upper part consists of 11 valve plates, the pressure drops on which are measured using PDI31015, PDI31003 and PDI31004. In the case of a high pressure differential, plates may be blocked or foamed in the absorber. In order to prevent foaming, the antifoam injection points are located on the supply line of the regenerated amine in the column to the flow regulators: FIC31004 and FIC31005. The output of saturated dietalomine to the F-303 receiver is regulated by the LIC31005 regulator.

Next, the purified gas passes through an F-319 separator, where about $99 \%$ of the amine solution is removed in droplet particles of
10 microns and more in size and discharged to F-303. The output of the amine solution in F-303 is controlled by the LIC31021 level controller. Sulfured gas from F-319 is directed to the suction line of the second stage of GC-201. The consumption of diethanolamine in the upper section is regulated by the flow regulator FIC31004, and in the lower section by the FIC31005. The consumption of purified gas from D-302 is measured using FI31003, the concentration of $\mathrm{H}_{2} \mathrm{~S}$ is measured by QRAH31001. A total of 457 sensors are located on the U300, information from which is collected on the basis of the distributed Honeywell DCS control system. Sensor specifications are presented in Table 3.

As an example, let consider daily measurements from the U300 medium-pressure gas cleaning unit. A fragment of the parameters database is presented in Table 4 .

\subsection{Simulation of the developed Smart technology on the example of a complex object of the medium pressure absorber}

Modern distributed control systems, such as the Honeywell DCS during the operation collect a huge amount of production data, the processing of which in industrial operation conditions is a complex task. Particular attention is paid to industrial safety, in connection with which there are different priorities of the alarms: emergency, high, low, logging and sending to the printer, writing to the log, sending to the printer and no action [21]. In this case, there are three alarms states: on, off, disabled. Therefore, for the application of the developed Smart technology there are selected 5 classes, depending on the state of the object for an alarm:

1 class. The alarm priority - emergency, the alarm state is on;

2 class. The alarm priority - high, the alarm state is on; 3 class. The alarm priority - low, the alarm state is off; 
G.A. Samigulina et al. / Advances in Science, Technology and Engineering Systems Journal Vol. 4, No. 3, $75-87$ (2019)

Table 3: Specification of sensors for the U300 circuit fragment, medium pressure gas cleaning block

\begin{tabular}{|c|c|c|c|c|c|}
\hline № & $\begin{array}{c}\text { Number of } \\
\text { position }\end{array}$ & Purpose of the device & Measure & $\begin{array}{l}\text { Measurement } \\
\text { range }\end{array}$ & Manufacturer \\
\hline D1 & LIC31053 & buoy level gauge & $\mathrm{kg} / 1007$ мм & $0,046-0,75$ & Fisher 2390B-249BF \\
\hline D2 & LIC31051 & buoy level gauge & $\mathrm{kg} / 559$ мм & $0,051-0,843$ & Fisher DLC3010-249BF \\
\hline D3 & LIC31052 & buoy level gauge & $\mathrm{kg} / 631 \mathrm{Mм}$ & $0,058-0,949$ & Fisher 2390B-249BF \\
\hline D4 & LIC31021 & buoy level gauge & $\mathrm{kg} / 500$ мм & $0,029-1,054$ & Fisher 2390B-249BF \\
\hline$\overline{D 5}$ & LIC31004 & buoy level gauge & $\mathrm{kg} / 500 \mathrm{MM}$ & $0,034-0,724$ & Fisher 2390B-249BF \\
\hline$\overline{D 6}$ & LIC31005 & difference converter & mbar & $0-294,2$ & Rosemount 3051CD2 \\
\hline D7 & $T 131050$ & temperature converter & ${ }^{\circ} \mathrm{C}$ & $0-100$ & $\begin{array}{l}\text { Honeywell STT350-0- } \\
\text { EP0 }\end{array}$ \\
\hline$\overline{D 8}$ & $P D I 31003$ & pressure drop & mbar & $0-280$ & Rosemount 3051CD3A \\
\hline$\overline{D 9}$ & PDI31004 & pressure drop & mbar & $0-180$ & Rosemount 3051CD2A \\
\hline D10 & FIC31004 & consumption regulator with indicator & mbar & $0-600$ & Rosemount G1151DP4E \\
\hline D11 & FIC 31005 & consumption regulator with indicator & mbar & $0-1124$ & Rosemount G1151DP5E \\
\hline D12 & QRAH31001 & analyzer of $\mathrm{H}_{2} \mathrm{~S}$ in gas & ppm $H_{2} S$ & $0-50$ & Tracor Atlas $722 \mathrm{R} / 102$ \\
\hline$\overline{D n}$ & $\ldots$ & $\ldots$ & $\ldots$ & $\ldots$ & ... \\
\hline
\end{tabular}

Table 4: Fragment of the parameters database of medium pressure gas purification process of $\mathrm{U} 300$

\begin{tabular}{|c|c|c|c|c|c|}
\hline № & TI31050 & LIC31053 & PDI31003 & ... & QRAH31001 \\
\hline 1 & 85,563 & 41,815 & 229,829 & .. & 44,509 \\
\hline 2 & 57,315 & 7,017 & 243,282 &.. & 43,887 \\
\hline 3 & 96,944 & 92,453 & 186,916 & .. & 47,597 \\
\hline 4 & 85,257 & 44,324 & 232,445 &.. & 237,979 \\
\hline 5 & 85,844 & 47,465 & 233,306 &.. & 46,529 \\
\hline 6 & 87,093 & 50,434 & 247,162 &.. & 45,659 \\
\hline 7 & 83,714 & 33,813 & 215,779 & $\ldots$. & 49,894 \\
\hline 8 & 86,189 & 40,041 & 228,184 &.. & 46,105 \\
\hline 9 & 85,688 & 41,387 & 228,406 &.. & 49,168 \\
\hline 10 & 83,381 & 32,606 & 213,857 &.. & 45,197 \\
\hline 11 & 82,75 & 31,564 & 212,562 &.. & 44,564 \\
\hline 12 & 84,76 & 30,187 & 211,865 &.. & 43,765 \\
\hline$\ldots$ & $\ldots$ & $\ldots$ & $\ldots$ &.. & $\ldots$ \\
\hline 800 & 74,379 & 51,453 & 220,456 &.. & 25,648 \\
\hline
\end{tabular}

4 class. The alarm priority - writing to the log and sending to the printer, the state of the alarm - prohibited;

5 class. The alarm priority - no action, the state of the alarm is prohibited.

www.astesj.com
Remark. The creation of classical mathematical models for complex control objects is a time consuming task. As a mathematical model for research, there is considered a time series consisting of records from the medium-pressure absorber sensors.

As a modeling environment, modern Rapid Miner software products and WEKA operators were used. Let consider a fragment of the DB_D302 database describing the state of the complex control object of the absorber D-302 at medium pressure gases cleaning on the U300 consisting of 12,000 copies of data. Figure 6 shows the visualization of results from the DB_D302 database sensors According to the $4^{\text {th }}$ algorithm, the database DB_D302 is pre-processed.

Then, within the framework of the multi-algorithm approach, the PCA algorithm is applied in order to reduce the lowinformative features that characterize the behavior of the complex control object $D$-302. Figure 7 shows the simulation results in the form of a graph of attributes ranking by degree of importance, as well as the visualization of the $D B \_D 302$ database after the noninformative parameters reduction. Similarly, the reduction of lowinformation descriptors is performed using the Random Forest algorithm (Figure 8) and the PSO swarm intelligence algorithm (Figure 9). 

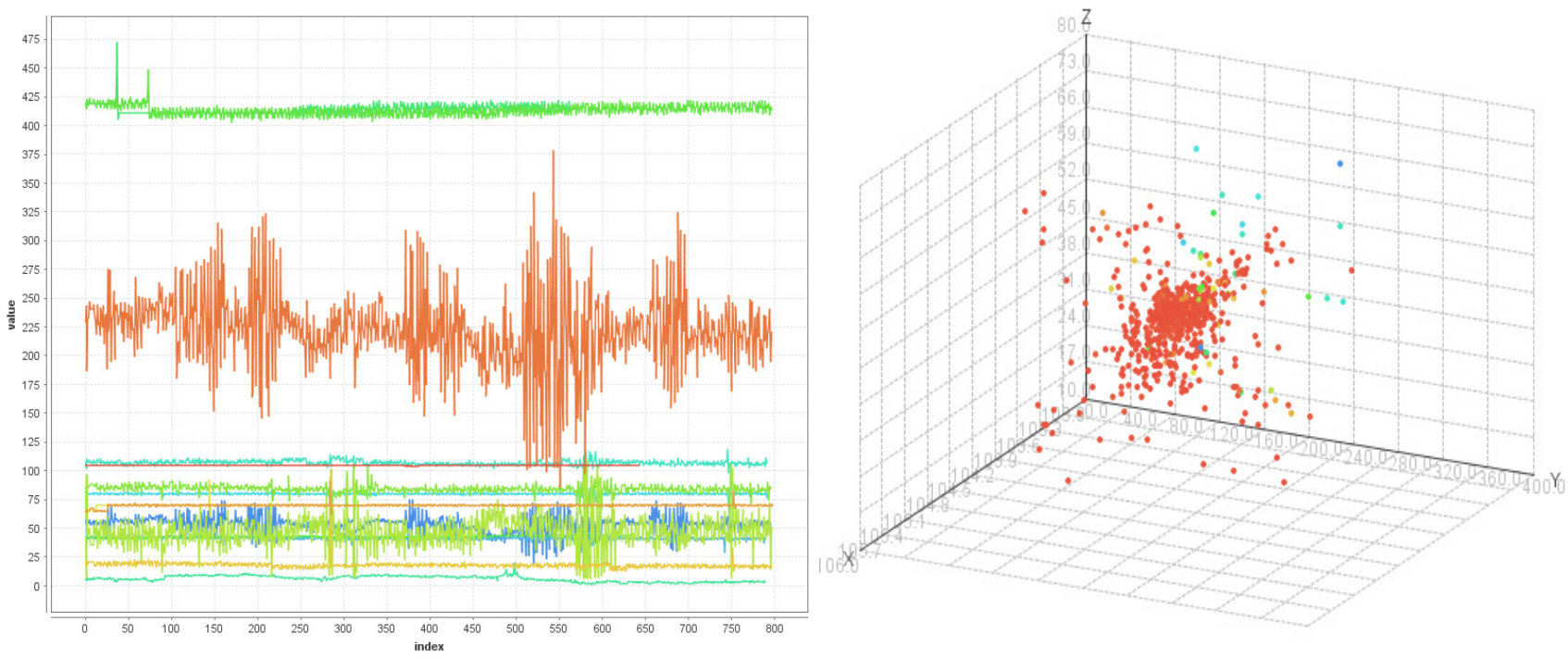

Figure 6: Fragment of $D B \_U 300 \_D 302$ database visualization in 2D and 3D form
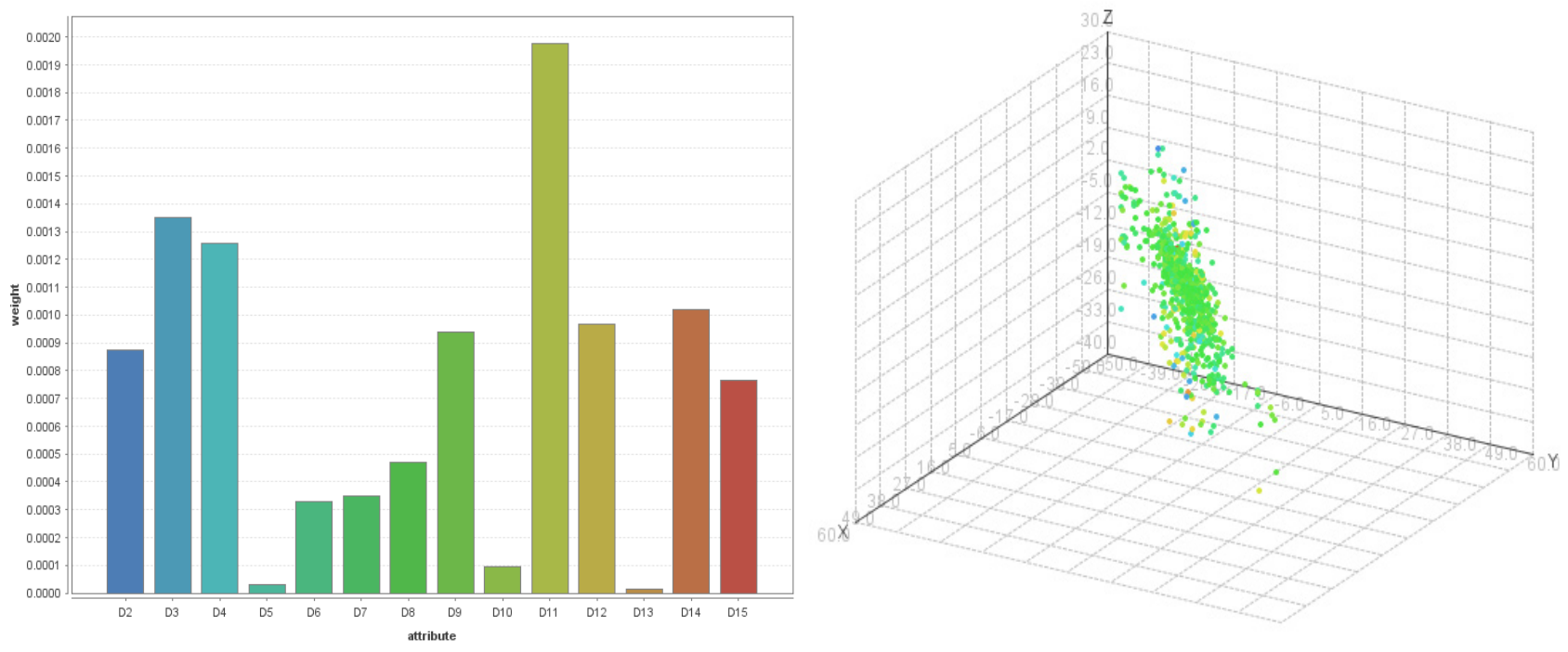

Figure 7: Low-informative parameters reduction using PCA
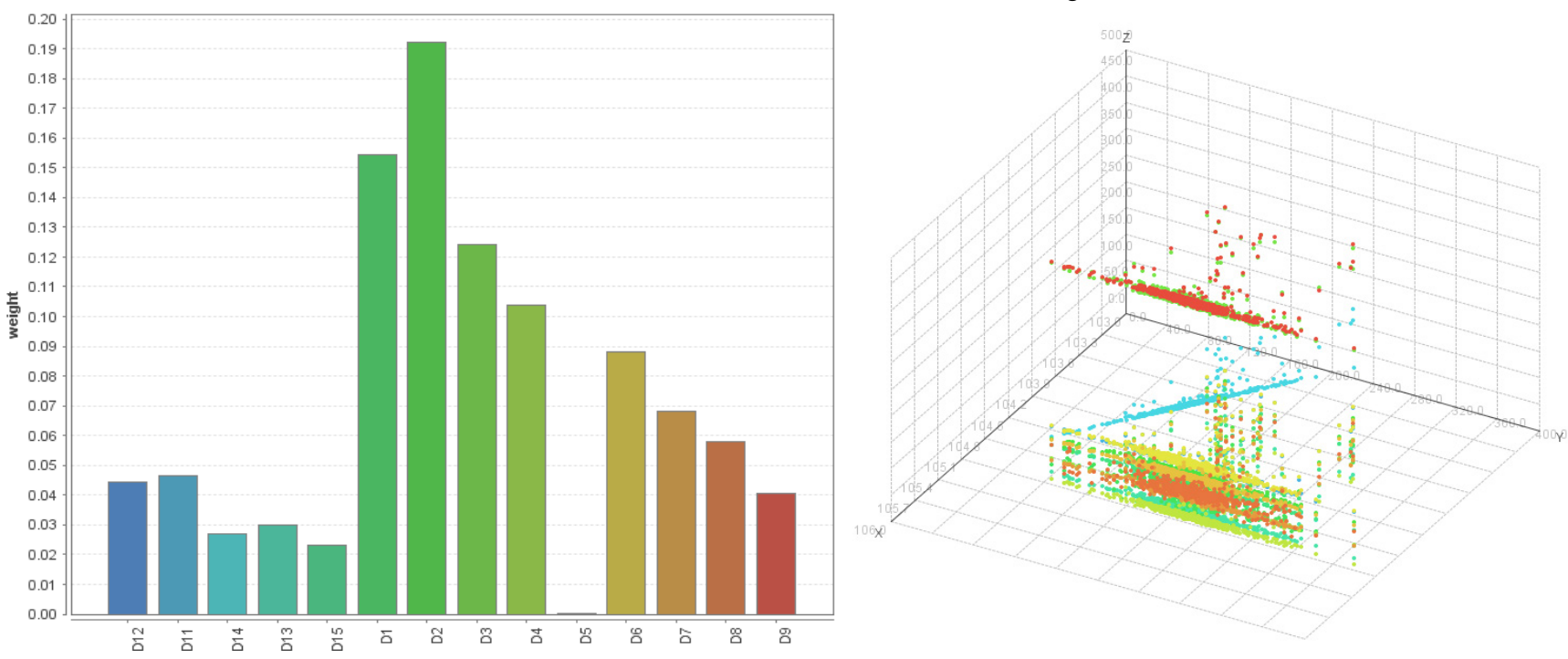

Figure 8. Low-informative parameters reduction using Random Forest 

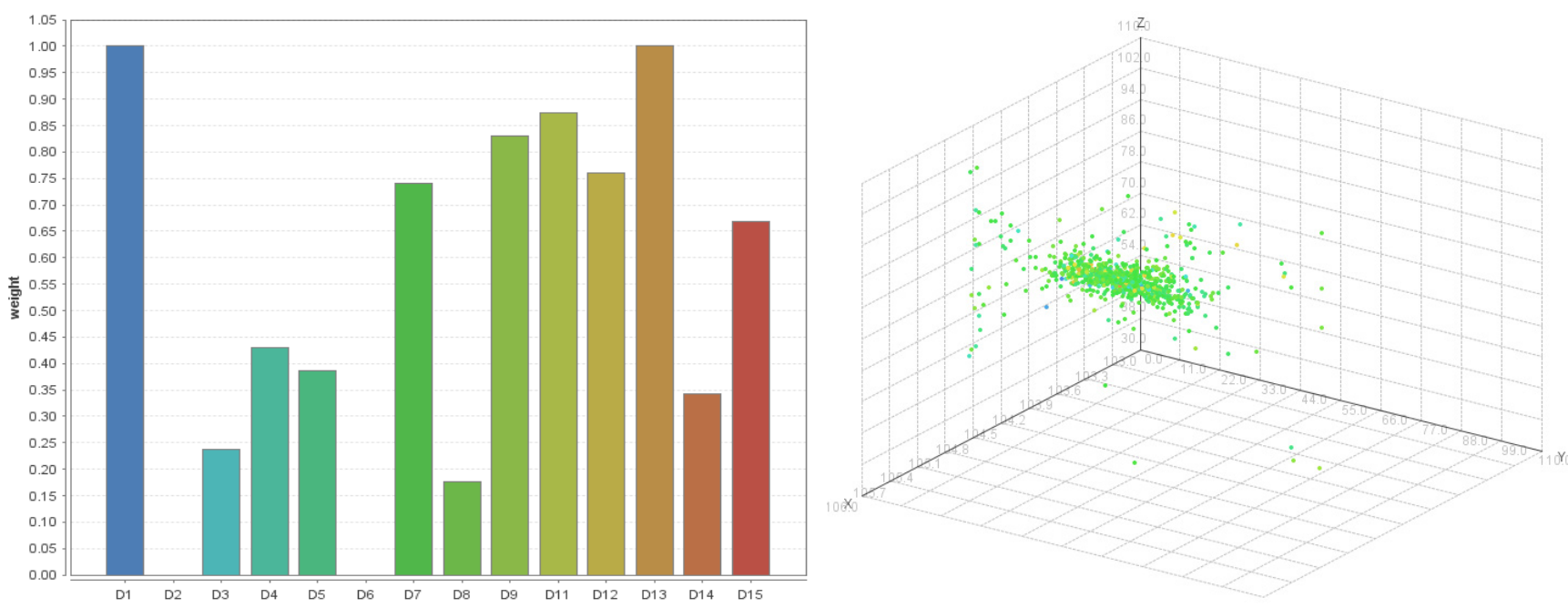

Figure 9. Low-informative parameters reduction using PSO

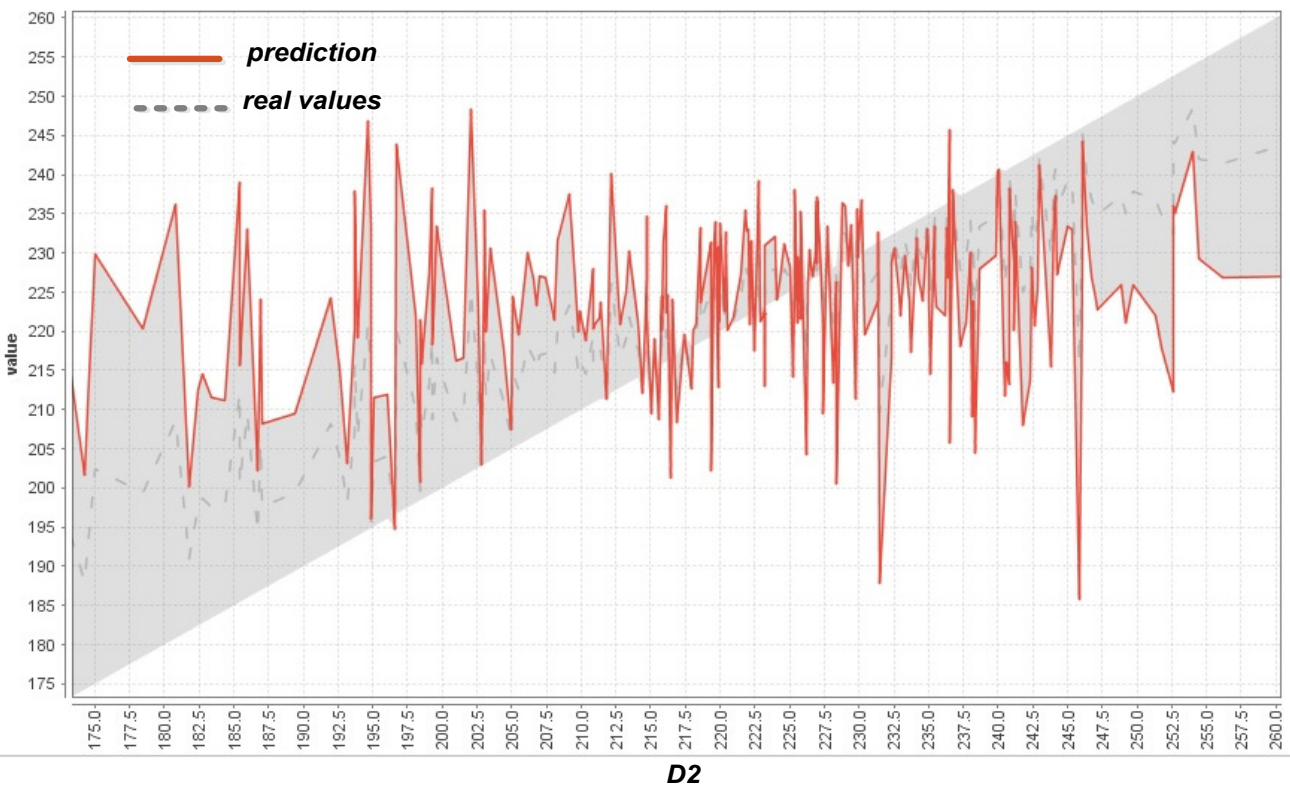

Figure 10. Prediction based on modified algorithm PSO - Clonal Selection

Further, according to the $4^{\text {th }}$ algorithm, after the selection of informative features, the task of classification is solved using the AIS algorithm, based on clonal selection. A comparative analysis of the simulation results is presented in Table 5 .

Table 5: Comparative analysis of image recognition results based on clonal selection

\begin{tabular}{|l|l|l|l|}
\hline $\begin{array}{l}\text { Feature selection } \\
\text { method }\end{array}$ & PCA & RF & PSO \\
\hline $\begin{array}{l}\text { Performance of feature } \\
\text { selection }\end{array}$ & $87,67 \%$ & $95,45 \%$ & $94,56 \%$ \\
\hline $\begin{array}{l}\text { Runtime of feature } \\
\text { selection }\end{array}$ & $1 \mathrm{~min} 25 \mathrm{sec}$ & $7 \mathrm{~min} 34 \mathrm{sec}$ & $1 \mathrm{~min} 4 \mathrm{sec}$ \\
\hline $\begin{array}{l}\text { Performance of clonal } \\
\text { selection classification }\end{array}$ & $85,53 \%$ & $86,74 \%$ & $93,67 \%$ \\
\hline $\begin{array}{l}\text { Runtime of feature } \\
\text { selection }\end{array}$ & $56 \mathrm{sec}$ & $2 \mathrm{~min} 59 \mathrm{sec}$ & $34 \mathrm{sec}$ \\
\hline
\end{tabular}

Because the Honeywell DCS distributed control system from the Installation 300 collects a huge amount of production data, the required time to simulate multidimensional data is a necessary criterion for evaluating the effectiveness of the modified algorithm.

In the case of the use of intelligent algorithms in the development of control systems, it is impossible to use classical sustainability criteria in order to assess the effectiveness of the developed Smart technology. The effectiveness of the heuristic algorithms is carried out using the calculation of such parameters as [35]:

- accuracy - accuracy (relative amount of correctly classified examples, percentage of correct predictions);

- classification_error - the percentage of incorrect predictions;

- $\quad k a p p a-$ kappa statistics calculation; 


\section{G.A. Samigulina et al. / Advances in Science, Technology and Engineering Systems Journal Vol. 4, No. 3, $75-87$ (2019)}

- weighted_mean_precision - weighted average value for each class of measurement accuracy;

- spearman_rho - rank correlation between actual and predictable labels using Spearman's rho measure, which shows a linear relationship between two variables;

- kendall_tau -rank correlation using Kendall's tau measure, showing the strength of the relationship between two variables;

- absolute_error - average absolute deviation of the predicted values from the actual value;

- relative_error - average absolute deviation of the forecast from the actual value divided by the actual value;

- relative_error_lenient - average relative error, which shows the average absolute deviation of the forecast from the actual value divided by the maximum of the actual value and the forecast;

- relative_error_strict - average severe relative error;

- normalized_absolute_error - normalized absolute error;

- root-relative-squared error - averaged relative quadratic error, etc.

Table 5 shows the final accuracy parameter showing the percentage of correct predictions by the proposed methods.

Therefore, according to the research results within the multialgorithm approach, the best prognostic result for solving the problem of prediction and control of the process of mediumpressure gases cleaning based on real data of the control object D302 is a modified algorithm PSO - Clonal Selection. A visualization of a fragment of the prediction results based on the PSO - Clonal Selection algorithm is presented on Figure 10.

\section{Conclusion}

As a result of the research, there was proposed a Smart technology for complex objects control based on Honeywell's distributed control system and an artificial immune system approach. The scientific novelty of the proposed technology is following:

1. The technology allows the prediction and control of complex objects of the oil and gas industry based on the intellectual analysis of historical data obtained from the distributed control system of Honeywell DCS.

2. There was developed a modified algorithm of artificial immune systems on the basis of a multi-algorithm approach in order to improve the quality of prediction of the behavior of complex objects under industrial operation.

3. There has been created a multi-agent Smart system that allows the integration of the proposed modified artificial intelligence algorithms into real industrial control systems.

4. Presented the simulation results on the basis of a real complex facility of the TengizShevroil enterprise (medium pressure absorber) of a fragment of the installation line 300 for gas cleaning from acid components.
Therefore, the proposed Smart technology allows the implementation of artificial intelligence algorithms based on real industrial production, in case when the classical methods of control theory are not efficient and unsuitable for working with large amount of data.

\section{Conflict of Interest}

The authors declare no conflict of interest.

\section{Acknowledgment}

The work was carried out under the grant of the KN MES of the Republic of Kazakhstan on the topic: AP05130018 "Development of cognitive Smart-technology for intellectual complex objects control systems based on artificial intelligence approaches" (2018-2020).

\section{References}

[1] D. O'Brien, "Smart operations", Pulp and Paper Canada, 2018.

[2] D. Yongsheng, Z. Lei, H. Kuangrong, Bio-Inspired clloaborative intelligent control and optimization, Springer, 2018.

[3] R. Hernandez, M. Lopez-Franco, E. Sanchez, A. Alanis, J. Ruz-Hernandez, Decentralized neural control, application to robotics, Springer, 2017.

[4] T. Wang, H. Gao, J. Qiu, "A combained adaptive neural network and nonlinear model predictive control for multirate networked industrial process control" IEEE Transactions on Neural Networks and Learning Systems, 27(2), 416-425, 2016. http://doi.org/10.1109/TNNLS.2015.2411671

[5] G. Yan, H. Fang, X. Chen, L. Shi, "Process control parameters optimal selection based on particle swarm algorithm", in $8^{\text {th }}$ International Symposium on Computational Intelligence and Design, Hangzhou, China, 2015. http://doi.org/10.1109/ISCID.2015.195

[6] K. Lakshami Narayana, V. Naveen Kumar, M. Dhivya, R. Prejila Raj, "Application of ant colony optimization in tuning a PID controller to a conical tank", 8(s2), 217-223, 2015. http://doi.org/10.17485/ijst/2015/v8iS2/61635

[7] A. Ramzy, A. Aldair, A. Almousawi, "Design an optimal PID controller using artificial bee colony and genetic algorithm for autonomous mobile robot", International Journal of Computer Applications, 100(16), 8-16, 2014. http://doi.org/10.5120/17607-8016

[8] K. Goher, S. Fadlallah, "Bacterial foraging-optimized PID control of a twowheeled machine with a two-directional handling mechanism", Robotics and Biomimetics, 4(1), 2-19, 2017. http://doi.org/10.1186/s40638-017-0057-3.

[9] R. Precup, R. David, E. Petriu, "Grey wolf optimizer algorithm - based tuning of fuzzy control systems with reduced parametric sensitivity", IEEE Transactions on Industrial Electronics, 64(1), 527-534, 2017. http://doi.org/10.1109/TIE.2016.2607698

[10] G. Ponticelli, S. Guarino, V. Tagliaferri, O. Giannini, "An optimized fuzzygenetic algorithm for metal foam manufacturing process control", The International Journal of Advanced Manufacturing Technology, 1-12, 2018. http://doi.org/ 10.1007/s00170-018-2942-5

[11] S. Mustapha, K. Faycal, S. Mohammed, "Application of artificial immune algorithm-based optimisation in tuning a PID controller for nonlinear systems", Int. J. Automation and Control, 9(3), 186-200, 2015. http://doi.org/ 10.1504/IJAAC.2015.070955

[12] Y. Peng, X. Luo, W. Wei, "A new control method based on artificial immune adaptive strategy", Electronika ir elektrotechnika, 19(4), 3-8, 2013. http://doi.org/10.5755/j01.eee.19.4.1246

[13] J. Daudi, "An overview of application of artificial immune system in swarm robotic systems", Advances in Robotics \& Automation, 4(127), 1-6, 2015. http://doi.org/10.4172/2168-9695.1000127

[14] H. Rezende, M. Silva, M. Santos, L. Honório, L. Silva, V. Vidal, J. Ribeiro, A. Cerqueira, A. Pancoti, B. Reginax, "Signal Estimation for UAV Control Loop Identification Using Artificial Immune Systems", in 22nd International conference on system theory, control and computing, 579-584, 2018. http://doi.org/ 10.1109/ICSTCC.2018.8540706

[15] F. Yao, F. Wang, M. Zhang, "Weak thruster fault detection for autonomous underwater vehicle based on artificial immune and signal pre-processing", Advances in mechanical engineering, 10(2), 1-13, 2018. https://doi.org/10.1177/1687814018758739

[16] J. Marciniak, K. Wawryn, P. Widulinski, “An Artificial Immune Negative Selection Algorithm to Control Water Temperature in the Outlet of the Chamber", in International Conference on Signals and Electronic Systems, 236-241, 2018. https://doi.org/10.1109/ICSES.2018.8507293

[17] Y. Ding, Nan Xu, S. Dai, L. Ren, K. Hao, B. Huang, "An immune system inspired reconfigurable controller", IEEE Transactions on Control Systems 
Technology, 24(5), $1875 \quad-\quad$ 1882, 2016. https://doi.org/10.1109/TCST.2016.2514847

[18] L. Puggini, J. Doyle, S. McLoone, "Fault detection using random forest similarity distance", IFAC-PapersOnLine, 48(21), 583-588, 2015. https://doi.org/10.1016/j.ifacol.2015.09.589

[19] K. Hanson, S. Yella, M. Doughert, H. Fleyeh, "Machine learning Algorithms in Heavy Process Manufacturing", American Journal of Intelligent Systems, 6(1), 1-13, 2016. https://doi.org/10.5923/j.ajis.20160601.01

[20] L. Yang, J. Zhang, F. Deng, J. Chen, "Sensor fault diagnosis based on on-line random forests", in 35th Chinese Control Conference, 1934-1768, 2016. https://doi.org/10.1109/ChiCC.2016.7553992

[21] Technological regulations for the technological process of hydrocarbon gases cleaning at the plant 300. TengizShevroil LLP, 2016.

[22] J. Brownlee, "Clonal selection algorithms", CIS Technical Report 070209A, 2007, 1-13.

[23] L. de Castro, F. von Zuben, "The Clonal Selection Algorithm with Engineering Applications", Proceedings of Workshop on Artificial Immune Systems and Their Applications, 36-37, 2000.

[24] U. Berna, S. Kulturel-Konak, "A review of clonal selection algorithm and its applications," Artificial Intelligence Review, Springer, 36(2), 117-138, 2011. https://doi.org/10.1007/s10462-011-9206-1

[25] J. Brownlee, "Clever Algorithms, Nature-Inspired Programming Recipes", 2011.

[26] J. Kennedy and R.C. Eberhard, "Particle swarm optimization", in IEEE International Conference on Neural Networks, 4, 1942-1948, 1995. https://doi.org/10.1109/ICNN.1995.488968

[27] J. Blondin, "Particle Swarm Optimization Applications in Parameterization of Classifiers", Armstrong Atlantic State University, 1-33.

[28] G.A. Samigulina, Z.I. Samigulina, "Modified immune network algorithm based on the Random Forest approach for the complex objects control", Artificial Intelligence Review, 2018. https://doi.org/10.1007/s10462-0189621-7

[29] L. Breiman, A. Cutler, "Random Forest", Berkeley, 2005.

[30] S.P. Chistyakov, "Random forests: a review", in proceedings of the Karelian Scientific Center of the Russian Academy of Sciences, 1, 117-136, 2013.

[31] L. Salazar, F. Mayer, D. Schütz, B.Vogel-Heuser, "Platform Independent Multi-Agent System for Robust Networks of Production Systems", IFAC Proceedings Volumes, 51(11):1261-1268, 2018. https://doi.org/ 10.1016/j.ifacol.2018.08.359.

[32] A. Sayed, S. Hanafi, A. Hassanien, "Multi-agent Artificial Immune System for Network Intrusion Detection and Classification", Proceeding of International Joint Conference SOCO'14, 145-154.

[33] S. German, S. Shin, A. Tsourdos, "Immune-system inspired approach for decentralized multi-agent control," in IEEE 24th Mediterranean Conference on Control and Automation (MED), 1020 - 1025, 2016. https://doi.org/10.1109/MED.2016.7536048

[34] G.A. Samigulina, Zh.A. Massimkanova, "Multi-agent system for forecasting based on modified algorithms of swarm intelligence and immune network modeling," in 12th International Conference Agents and Multi-agent Systems: Technologies and Applications (KES-AMSTA-18), 199-208, 2018. https://doi.org/10.1007/978-3-319-92031-3 19

[35] M. Hofmann, R. Klinkenberg, "RapidMiner: Data Mining Use Cases and Business Analytics Applications", 2014, 48-49. 